\title{
Multichannel continuous flow analysis on the SMA-4/-7A
}

\author{
M. G. NELSON \\ From the Department of Clinical Pathology, Royal Victoria Hospital, Belfast
}

The exponential rise in laboratory requests has made it essential to develop scientific equipment capable of processing more efficiently the large number of repetitive tests. For this purpose, instruments which operate by continuous flow analysis have proved to be eminently successful.

Although used primarily in departments of clinical biochemistry, they are also useful in diagnostic haematology in the single-channel mode for haemoglobinometry, serum iron measurement, and antibody screening, and in the multichannel mode for blood group determination and for routine haematological parameters.

We have already reported (Nelson and Lamont, 1961) how the introduction of a single channel AutoAnalyzer for haemoglobinometry increased productivity when measured in terms of numbers of tests performed per technician per year. However, subsequent studies indicated that something of the order of half of the total work load consisted of four tests, namely, the determination of the haemoglobin, the haematocrit, and the total red and white cell counts. Thus while mechanization of individual tests may make a significant contribution to efficiency, the greatest benefit is likely to be achieved by the 'automation' of the four basic tests. The SMA-4/-7 is a commercially available item of multichannel equipment capable of determining these four basic parameters from a single sample of blood.

\section{THE EQUIPMENT}

This equipment consists of the following modules: a sampler, a proportioning pump with plastic tubing manifolds and glass helical mixing and phasing coils, a conductivity flow cell, a tubular flow cell colorimeter, a dual channel cell counter, a programmer, and a single pen recorder. The anticoagulated whole blood in the sample cup is mixed by twin paddles and an aliquot taken up by the sampler crook. This is then split by the 'sample splitter' into four streams which flow along the plastic tubing manifolds, mixed with appropriate $\stackrel{\text { in }}{N}$ reagents and passed via phasing coils to individual $\omega$ detecting devices for the haematocrit, haemo- $\overrightarrow{\mid}$ globin, and cell count determinations. The output 0 from each of the detectors is monitored by the 음 programmer and fed sequentially to a single pen recorder. An SMA-4 can be readily converted into a -7 by the addition of a calculating element to the programmer which determines the $\mathrm{MCV}, \mathrm{MCH}, \stackrel{2}{2}$ and MCHC. The instrument operates at a rate of 60 samples per hour and the results are $\mathscr{\mathscr { \sigma }}$ graphically recorded and immediately readable.

Using the original manifold we found that the results obtained in the red cell count channel gave a coefficient of variation which ranged up to $5 \%$. This was considered to be unsatisfactory and found to be due, in large measure, to irregular pumping along large diameter tubes which tended to lose their elasticity. Consequently, the manifold was redesigned and each large diameter tube was replaced by a number of tubes of narrower bore. The Technicon Company have introduced a considerable number of improvements in the equipment which is now marketed as the SMA-4A or 7A.

In the second generation machines the manifold 8 has been extensively modified and no tubing has a bore greater than $0.073 \mathrm{in}$. It is claimed that this 을 has resulted, among other things, in a more constant flow rate, reduced reagent consumption, reduced wear on the tubes and, at the same time, a reduced rate of flowline blockage. Apart from changes in the manifold other improvements in the new $N$ 'A' range include alterations in the optics of the cell $\mathbb{\omega}^{N}$ counter, in the electronics of the programmer, and in $\sigma$ the recorder where the output of all the channels 0 has been linearized.

\section{THE HAEMATOCRIT}

In the SMA-4/-7 the haematocrit is derived from the electrical conductivity of the anticoagulated whole $\stackrel{\mathbb{Q}}{\Omega}$ blood sample as it passes through a conductance chamber. Using such a detecting device a number of 


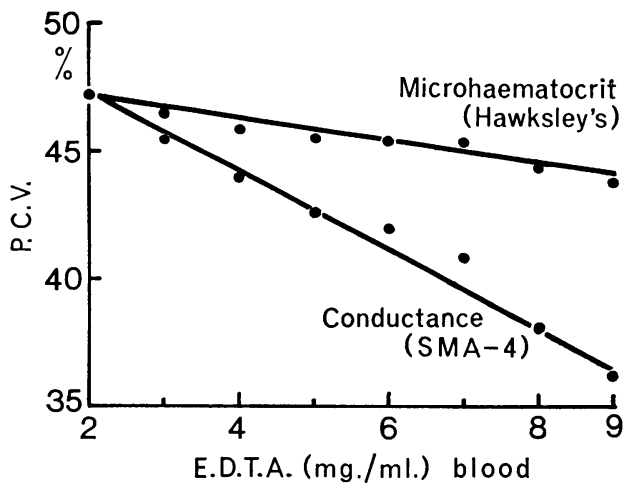

FIG. 1. Effect of concentration of EDTA anticoagulant on the PCV determination.

factors could be operative apart from the relative volume of red cells in the suspending medium.

In the first place electrolytes derived from the anticoagulant in the sample could be responsible for a proportion of erratic results. The depressing effect of excess anticoagulant on the haematocrit when determined by centrifugation is shown in Figure 1. When, however, the haematocrit is derived from conductivity measurement, the excess anticoagulant has a much more significant effect. This adverse effect can be virtually eliminated by ensuring that all specimen bottles are adequately filled with blood. Furthermore, discrepancies caused by minor variations in the filling of specimen bottles can be made less significant by reducing the concentration of EDTA from 2 to $1.6 \mathrm{mg}$ per $\mathrm{ml}$. This latter concentration is adequate for anticoagulant purposes and in practice does not result in any measurable increase in the number of clotted specimens.

Other causes of error in the haematocrit channel have been investigated by Davis, Bresland, and Green (1966) who reported that 'variation in plasma electrolytes up to $150 \mathrm{~m}$-equiv/l, leucocyte counts up to 30,000 per $\mathrm{cmm}$, and protein concentration up to 9 grams per cent did not influence the results'. Davis et al also reported that $86 \%$ of 500 samples gave results which were within $\pm 2 \%$ of the microhaematocrit centrifugation method.

\section{SCIENTIFIC EVALUATION OF THE SMA-4/-7A}

Scientific evaluation of this multichannel haematological equipment started in July 1967 and experience with the modified SMA-4has extended over 18 months. This included a six-month period of special investigation on the test bench followed by a year in the routine service laboratory, and the results have just been published (Lappin, Lamont, and Nelson, 1969). The second generation machine, the 7A, was only delivered a few weeks ago, so that it has not yet been subjected to an exhaustive trial.

The test schedule has been designed to obtain information on the following points: (1) the amount of carryover from one sample to another; (2) the precision and accuracy of the results obtained on each of the four channels; (3) the rate of throughput of test specimens under working conditions; (4) the mechanical and electronic reliability; (5) the problems of data handling; and (6) the economic cost.

CARRYOVER In a continuous flow system it is possible for discrepancies to arise due to crosscontamination mainly at the point of sampling. We have measured the amount of carryover from a radioiodinated serum albumin sample upon a blank immediately following and the degree of dilution of the labelled sample by a blank preceding it. By this technique the carryover in the SMA-4 was found to be less than $1.5 \%$.

The use of labelled serum albumin to test carryover has been criticized, quite rightly, on the grounds that it does not use the same sample material as the equipment, namely, whole blood. Using the same technique as before, we have tested the 7A with whole blood in which the erythrocytes have been labelled with ${ }^{51} \mathrm{Cr}$ and the plasma with radioiodinated albumin. The total carryover in the new equipment was found to be less than $0.5 \%$. This very small carryover is due, in large measure, to a better design of the mixing paddles and to the fact that these are now treated with a water-repellent silicone coat.

PRECISION The precision was determined by repeated analysis of the same sample and from these results calculating the standard deviation and the coefficient of variation. In order to test the precision over as wide a range as possible, replicate determinations of a number of samples of differing concentration were carried out and the coefficient of variation from each set of observations was obtained. The results are presented in a summarized form for both the SMA-4 and -7A (Table I). Absolute precision in measuring the haematological parameters is difficult to attain and for diagnostic purposes may not be necessary. The degree of precision required for clinical use has never been stated but with modern equipment it is possible to reach and maintain at least the following standards of reproducibility, namely, haemoglobin $\pm 1 \%$, PCV $\pm 2 \%$, erythrocyte count $\pm 2.5 \%$, leucocyte count $\pm 5 \%$. Compared with these 
TABLE I

PRECISION OF THE SMA-4 AND -7A

\begin{tabular}{|c|c|c|c|c|}
\hline & \multicolumn{4}{|c|}{ Coefficient of Variation (\%) } \\
\hline & \multicolumn{2}{|c|}{ SMA-4 (Modified) } & \multicolumn{2}{|l|}{$S M A-7 A$} \\
\hline & Range & Mean & Range & Mean \\
\hline $\begin{array}{l}\text { Haemoglobin } \\
\text { Haematocrit } \\
\text { Red cell count } \\
\text { White cell count }\end{array}$ & $\begin{array}{l}0 \cdot 38-0.92 \\
0 \cdot 00-2 \cdot 08 \\
1 \cdot 51-3 \cdot 62 \\
1 \cdot 65-3 \cdot 23\end{array}$ & $\begin{array}{l}0.54 \\
0.83 \\
2 \cdot 37 \\
2 \cdot 43\end{array}$ & $\begin{array}{l}1 \cdot 05-3 \cdot 48 \\
0 \cdot 53-1 \cdot 17 \\
1 \cdot 72-3 \cdot 96 \\
0 \cdot 79-7 \cdot 35\end{array}$ & $\begin{array}{l}2 \cdot 11 \\
0 \cdot 97 \\
3 \cdot 10 \\
3 \cdot 26\end{array}$ \\
\hline
\end{tabular}

TABLE II

ACCURACY OF SMA-4 AND -7A

\begin{tabular}{|c|c|c|c|c|}
\hline & \multicolumn{2}{|l|}{$S M A-4$ (Modified) } & \multicolumn{2}{|l|}{$S M A-7 A$} \\
\hline & Correlation Coefficient & Regression Equation & Correlation Coefficient & Regression Equation \\
\hline $\begin{array}{l}\text { Haemoglobin } \\
\text { Haematocrit } \\
\text { Red cell count } \\
\text { White cell count }\end{array}$ & $\begin{array}{l}0.9942 \\
0.9668 \\
0.9583 \\
0.9905\end{array}$ & $\begin{array}{l}y=1.003 x-0.04 \\
y=0.921 x+3.41 \\
y=0.990 x+0.03 \\
y=1.003 x+0.15\end{array}$ & $\begin{array}{l}0.9904 \\
0.9546 \\
0.8695 \\
0.8740\end{array}$ & $\begin{array}{l}y=0.984 x+0.19 \\
y=0.986 x+0.98 \\
y=0.904 x+0.35 \\
y=0.942 x+0.35\end{array}$ \\
\hline
\end{tabular}

figures for precision, which were obtained using individual items of equipment, the results achieved on the multichannel machine are satisfactory.

ACCURACY In haematology the accuracy is not usually determined by measuring how close the observed value is to the 'true' value; instead it has to be derived from some method of mathematical computation. To this end 100 blood samples were divided into two aliquots and submitted to analysis on the SMA-4 and by acceptable standard methods.

Each pair of results was then subjected to statistical analysis by calculating the regression equation and the correlation coefficient. The results are presented in graphical form in four scattergraphs (Figs. 2-5). The general conclusion to be drawn is that for each of the parameters there is good correlation between the two methods of measurement. The results are summarized in Table II.

For three parameters the regression line on the scattergraphs intercepts the axis at or near zero, but for the haematocrit there would appear to be a positive intercept. However, the line derived from the regression equation was not continued below the lower limit of our measurement as there were no data in this area and it may not necessarily have been linear. Despite this anomaly the correlation between the haematocrit on the SMA-4 and the centrifugation micronhaematocrit method shows good agreement throughout the range which we studied.

QUALITY CONTROL We routinely use two independent methods. For one of these we prepare our red cell suspension as a secondary or working standard. This is a preserved blood cell suspension in which the erythrocytes in anticoagulant are preserved by the addition of adenine and inosine and such suspension is stable for at least four weeks. It is used to set the haemoglobin, the red cell count, and the haematocrit channels and to detect for instrumental drift by introducing it into the sample plate in every twentieth slot.

We also use the cumulative sum technique as a statistical method of quality control. For this the results of the haemoglobin and the haematocrit determinations are collected each day, the mean is determined, and the difference from the 'grand mean' is algebraically calculated and cumulatively plotted on a graph.

FREQUENCY OF UNACCEPTABLE RESULTS In any routine run of tests, a proportion of results could be 'unacceptable' because the data are either inadequate or inaccurate: For instance, with this equipment white cell counts above 20,000 on the SMA-4 or 25,000 on the SMA-7A, are not recorded and such results are therefore 'unacceptable' because they are incomplete. Another possibility of error arises when the blood is from a patient with dysproteinaemia which can lead to excessive rouleaux formation, a false red cell count, and haematocrit determination with incorrect derived indices.

Thus, even though an item of equipment has been calibrated and found to produce results which are both precise and accurate, and even though standards introduced at regular intervals are satisfactory, it does not necessarily follow that all the results throughout a run will be correct.

It is because incorrect results may occur sporadically that the use of a trained haematological tech- 

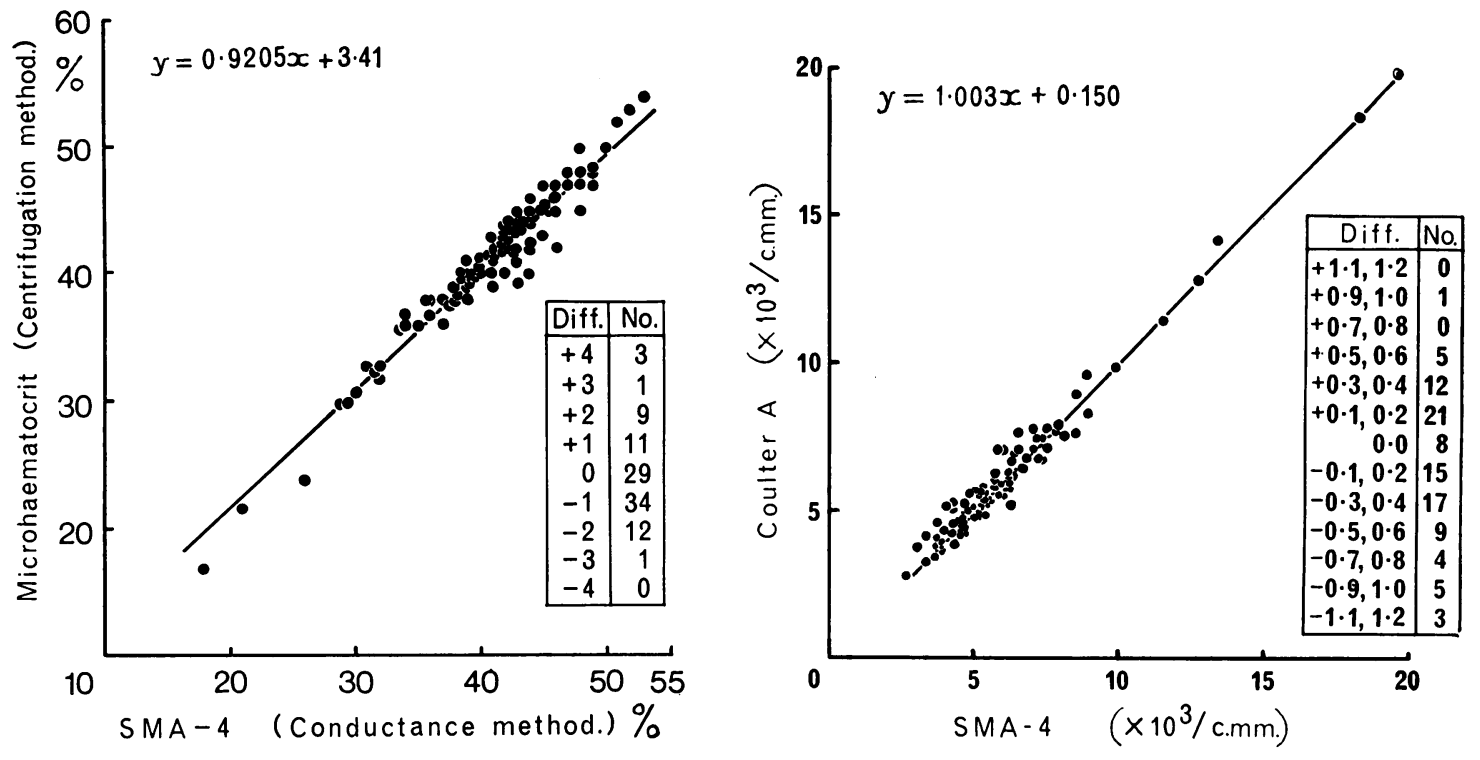

FIG. 2. Comparison of duplicate haematocrit determinations.
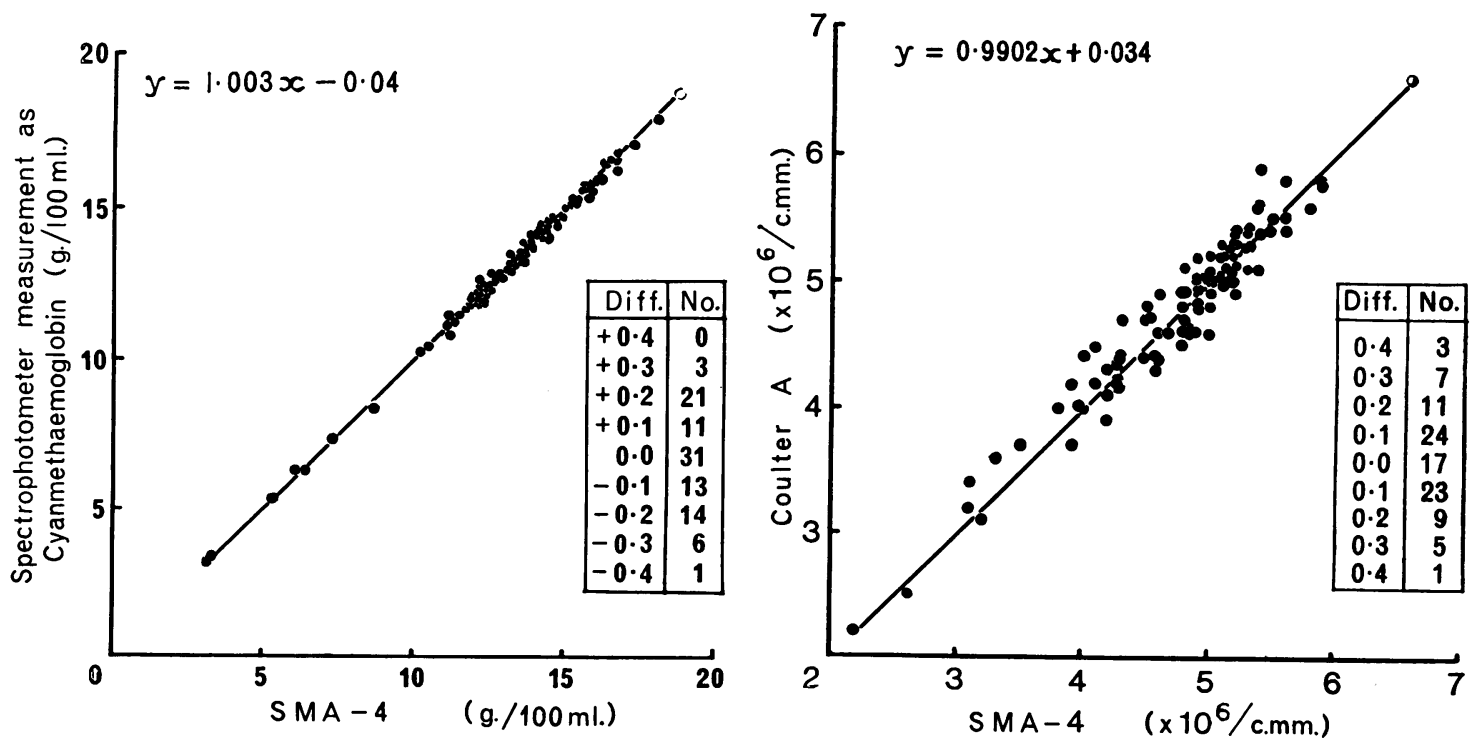

FIG. 4. Comparison of duplicate haemoglobin determinations.

FIG. 5. Comparison of duplicate erythrocyte determinations. 
nician as a machine operator is necessary. Such an operator can detect some obviously fallacious results from the indices.

Using indices beyond the normal range or 'lacking in fit' as markers we have determined the frequency of 'unacceptable' results, both during the special study and later during routine use. The frequency of 'unacceptable' results obtained on the SMA-4 would tend to indicate that while faults occur occasionally in the red cell count and haematocrit channels they are less frequent in the white cell count and haemoglobin channels (Table III).

SPEED OF OPERATION It requires about half an hour to calibrate and test the equipment. Although the normal operational speed is 60 samples per hour, because of the introduction of blanks and quality controls, the total number of test samples which can be passed through the machine in working conditions is of the order of 48 per hour. For most hospital laboratories this is a satisfactory rate of throughput and permits the analysis of at least 300 samples per working day. The number of specimens examined can, of course, be increased by extending the working day or by shift work.

MECHANICAL AND ELECTRONIC RELIABILITY I am in no doubt that continuous flow analysis is a scientific technology in its own right. Present equipment is far from foolproof and is definitely not yet at the press-button stage. The operator must have experience and interest in this type of equipment, and some knowledge of mechanics, hydraulics, and general biomedical engineering. There is a considerable advantage if such an operator can call on the immediate services of an electronic technician.

Although the original equipment was, for the most part, electronically stable it was subject to mechanical blockage at certain critical points. The number of such stoppages could be reduced but not entirely eliminated by strict attention to routine maintenance. Filters have now been fitted to the reagent and air flow lines so that, even when the new range of equipment is in constant routine use, mechanical blockage is rare.

The presence of dust in the environment can also have an adverse effect on the micro-switches of the programmer. As a result, preventive maintenance of the electronic components is needed in our laboratory at least once every two weeks.

If a more serious fault should occur this can be remedied after it has been diagnosed, and to help trace the new fault a new Troubleshooter's Manual is available. The replacement of the defective component is more readily achieved because of the modular construction of the apparatus and the good replacement and repair service.

DATA PROCESSING The graphical record of the results of the analyses, while it looks attractive, can be criticized on a number of grounds. In the first place the sheet of paper is so large that it does not readily fit existing medical record documents and furthermore the test information is not stored. As a consequence, concurrent recording directly from the chart on to a work sheet or some other storage document has been found to be necessary. This requires a trained haematological technician to observe the chart, to digitize and record the results and, if there is any obvious error, to submit the sample to test in some other way. As a consequence, it is necessary to employ a second part-time technician as an acolyte to fill the sampler plate and to carry out other menial tasks. The introduction of some data acquisition equipment would eliminate the need for manual recording and provide a new dimension in data handling. I have been given to understand that such equipment has now become available which would increase the accuracy of recording and reduce the labour component involved.

COST ANALYSIS A cost analysis of the SMA-4 for one year has been carried out. This has included the salaries and wages of the personnel, the cost of materials and reagents, the maintenance of the apparatus, and amortization of the equipment over a five-year period. The operational cost worked out at $£ 77.4$ per 1,000 samples or 1.55 shillings per sample. This study also showed that salaries and wages constituted $43 \%$ of the total and that amongst the materials the most expensive by far was the chart paper (Table IV).

TABLE III

PERCENTAGES OF UNACCEPTABLE RESULTS ON SMA-4 (MODIFIED)

\begin{tabular}{llll} 
& Special Study & Routine Use & Variation of Standard \\
\hline Haemoglobin & 0.0 & 0.9 & $15.4 \pm 0.2 \mathrm{~g} / 100 \mathrm{ml}$ \\
White cell count & 0.6 & 1.8 & - \\
Haematocrit & 4.0 & 5.1 & $49 \pm 1.0 \%$ \\
Red cell count & $1 \cdot 1$ & 7.0 & $5.2 \pm 0.1 \times 10^{\circ} \mathrm{cmm}$
\end{tabular}


TABLE IV

COST ANALYSIS OF SMA-4 IN 1968

Cost $\quad$ Total
$(£)$

(£)

\begin{tabular}{lrr}
\hline Staff & & \\
Trained technician & & \\
Full time \\
Part time & 1,500 & \\
& 500 & 2,000 \\
Materials & & \\
Reagents & 39 & \\
Manifolds & 94 & \\
Plastic cups & 80 & \\
Chart paper & 850 & 1,063 \\
Electricity & 50 & 50 \\
Maintenance and replacements & 93 & 93 \\
Amortization over five years & 1,440 & 1,440 \\
Total & & $£ 4,646$
\end{tabular}

If the output were digitized and reproduced in some record-compatible form, neither the expensive precalibrated draft paper nor the second operator would be needed. The resulting saving in revenue expenditure would be of such an order that it would prove adequate either to hire the data processing attachment or to amortize its capital cost.

\section{CONCLUSIONS}

At the present time the best contribution to efficiency in a hospital diagnostic haematological laboratory would be achieved by the 'automation' of the four basic tests, namely, the haemoglobin and haematocrit determinations and the erythrocyte and leucocyte counts. A number of so-called 'automatic' items of multichannel equipment are commercially available for this purpose.

The requirements of a fully automated laboratory analytical system should include at an economic cost, at least the following: a means of positive specimen identification, a self-loading mechanism for specimens, the uptake of a sample without carryover, the automatic performance of a series of operations, a detecting device for the end product, a servo-operated self-regulating feedback mechanism to control any instrumental drift, a means of visualizing or recording the result, linkage to a digitizer, and a reproducer of record-compatible data with backing store capability, the production of precise and accurate results at a satisfactory throughput rate, freedom from mechanical and electronic breakdown, and ease of operation by unskilled workers.

The Technicon SMA-4/-7A in the currently available form meets some but not all of these requirements. Nonetheless its routine use for more than a year in a hospital diagnostic service has shown its capacity to handle a work load of up to 300 specimens per working day and its acceptability by laboratory technicians. The precision and accuracy are within a satisfactory range. The cost has been assessed at 1.55 shillings per specimen for the SMA-4 and 1.64 shillings for the SMA-7A. The test results are visualized in graphical form and can be detached for use as a report document but no permanent record is produced. A data acquisition, digitizing, and printing attachment is currently being developed and should become available as an optional extra. Ideally, this accessory item of equipment should produce results in a form compatible with presently accepted hospital documentation systems and suitable both for storage as a permanent record and for input to a computer.

I wish to acknowledge my indebtedness to Mr A. Lamont and Mr T. R. J. Lappin for their skilled technical work, and to the Department of Medical Statistics, Queen's University, Belfast, for their advice and help with the planning and presentation of the scientific evaluation.

I am also grateful to the Editor and Publishers of the Journal of Clinical Pathology for permission to reproduce Figures 1 to 5.

\section{REFERENCES}

Davis, R. E., Bresland, R., and Green, R. (1966). Evaluation of electronically measured haematocrit values. Lab. Pract., 15, 1376.

Lappin, T. R. J., Lamont, A., and Nelson, M. G. (1969). An evaluation of the AutoAnalyzer SMA-4. J. clin. Path., 22, 11.

Nelson, M. G., and Lamont, A. (1961). Haemoglobinometry by an automatic analytical procedure. Ibid., 14, 448. 